Volume 9, No.3, May - June 2020

International Journal of Advanced Trends in Computer Science and Engineering

Available Online at http://www.warse.org/IJATCSE/static/pdf/file/ijatcse30932020.pdf

https://doi.org/10.30534/ijatcse/2020/30932020

\title{
Design of a Smart Medical Bracelet Prototype for COVID-19 based on Wireless Sensor Networks
}

\author{
Oussama Ghorbel ${ }^{1,2,3}$, Rami Ayedi ${ }^{1}$, Haithem Ben Chikha ${ }^{1}$, Ousama Shehin ${ }^{1}$, Mariem Frikha \\ ${ }^{1}$ Jouf University, Saudi Arabia, E-mail: oaghorbel@ju.edu.sa. \\ ${ }^{1}$ Jouf University, Saudi Arabia, E-mail: rayadi@ju.edu.sa. \\ ${ }^{1}$ Jouf University, Saudi Arabia, E-mail: orshahin@ju.edu.sa. \\ ${ }^{1}$ Jouf University, Saudi Arabia, E-mail: hbchikha@ju.edu.sa. \\ Department of Computer Science, E-mail: maryam.frikhaa@gmail.com. \\ ${ }^{2}$ National Engineers School of Sfax, Sfax University, Tunisia \\ ${ }^{3}$ Digital Research Center (CRNS), Technopark Sfax, Tunisia
}

\begin{abstract}
According to World Health Organization, Coronavirus (COVID-19) is the disease caused by the new corona virus that emerged in China in December 2019. In this proposal, we propose a research project idea of a Corona Virus symptoms using high-temperature body. This project is a part of early prevention and to help control the emerging Corona epidemic (COVID-19). The last decade has seen a proliferation of fields of application for wireless sensor networks (WSNs). One of these promising areas is health, where the wireless sensor networks (WSNs) can provide important support that makes it easier to monitor patients' vital signs and send alerts in real time. This will help medical personnel to intervene in a timely manner in the event of complications. In this paper, we present the hardware and software design of wireless sensor networks based Intelligent Medical Bracelet (IMB) for the measurement and storage of body temperature and heartbeat values. The IMB also allows three types of alert to be triggered if one of the vital signs is exceeded.
\end{abstract}

Key words : Wireless Sensor Networks (WSNs), Health, Smart Medical Bracelet, Prototype, Waspmote, BMI, Energy, Gateway.

\section{INTRODUCTION}

A wireless sensor networks (WSNs) is a network composed of sensor nodes integrating the acquisition, processing, and communication functionalities. Once deployed, the nodes cooperate with each other autonomously in order to collect and transmit data to a base station in order to monitor and / or control a given phenomenon [3].

Traditionally, the sensors as well as the monitoring unit are fixed for the patient by wire which induces a restriction of the movement of the patient. In addition, when the patient needs to be moved, all monitoring devices must be moved, which means that they should be disconnected and then reconnected. This brings overhead, wasted time and redundant work [11], [12]. Today, with the emergence of bio-telemetry systems and the enormous progress in wireless communication systems, real-time monitoring of patients can be ensured even if patients are far from the hospital. This allows an improvement in the quality of life of the patients, decreasing the patient's discomfort by extending the scope of this health care. In this document, we present the hardware and software design of a prototype of a Wireless Sensor Networks platform for health.

In 2020, the coronavirus pandemic known as (COVID_19), it threatens human lives, disrupting the economy and stopping people's daily life [15], [16], [17], [18]. This disease caused by the new coronavirus that.

Emerged in China. COVID-19 appeared in Wuhan, a city in China, in December 2019. The risk coronavirus or a similar pandemic might occur in the future is even higher for those who work in hospitals, or those who gather in public places [19], [20], [21], [22].

Although health officials are still tracing the exact source of this new coronavirus, early hypotheses thought it may be linked to a seafood market in Wuhan, China. Some people who visited the market developed viral pneumonia caused by the new coronavirus [23], [24], [25]. A study that came out on Mars.25, 2020, notes that the individual with the first reported case became ill on Dec. 1, 2019, and had no link to the seafood market. Most people infected with the COVID-19 virus will experience mild to moderate respiratory illness and recover without requiring special treatment. It appears that symptoms are showing up in people within 14 days of exposure to the virus. COVID-19 can be severe, and some cases have caused death [26], [27], [28], [29].

Until now, the confirmed cases are 465,915,721,031 confirmed deaths and 200 Countries, areas or territories with cases. At this time, there are no specific vaccines or treatments for COVID-19. However, there are many ongoing clinical trials evaluating potential treatments. 
The rest of this paper is organized as follows: we explore, in section 2, the state of the art of the various medical bracelet solutions proposed in the literature or on the market. Section 3 is devoted to the presentation of the general context of the work, the target population as well as the different functionalities of the Smart Medical Bracelet. The constraints to be taken into account when designing the Bracelet are presented in section 4. A description of the characteristic of Waspmote are presented in section 5. In section 6, we detail the methods and tools for the hardware and software design of the Bracelet. We end with a conclusion and a presentation of the work in progress.

\section{STATE OF THE ART OF MEDICAL BRACELETS}

Several researches are interested in the bio-telemetry field in order to remedy the problems of remote diagnosis. Among these projects, there are those who were involved in the manufacture of medical bracelet. Indeed, several products have been implemented.

For example, we find as a first solution the COLUMBA bracelet considered as an anti-fugue solution [2], [4], [6], [10]. This bracelet was developed in 2006 by the Canadian mobile tele-security company 'Medical Intelligence Technologies Inc'. This is one of the solutions proposed for the problems of running away and wandering caused by Alzheimer's disease. Combining a mobile phone, a GPS geo-location system and an intelligent alert system (detecting any exit from a safe area), the COLUMBA bracelet allows you to quickly locate the person who has it when he leaves a predefined area, thereby reducing the risks associated with wandering problems.

As a second solution we find "the automatic implantable defibrillator" [1], [7], [8], [9], It is a device which, after intracorporeal insertion, is capable of detecting and interrupting, by electric shock, a ventricular fibrillation responsible for 'cardiac arrest. This device allows you to control atrial functions, whether detection, stimulation and even defibrillation. A Holter function stores several cycles preceding the detection. It constitutes a database, dates critical cardiac events, confirms the proper functioning of the detection probe. This defibrillator is linked to a system that allows the implanted patient to benefit from remote monitoring and controls.

The third solution called Oxy-ped [5] is specially made for pediatric use. The sensor is designed to adapt to the finger of children from 2 years old. Oxy-ped measures oxygen saturation and heart rate on the patient's finger (adults) and displays the results on an LCD screen. It can be used in hospitals, medical centers, at home, before and after sports. It triggers an audible and visual alarm when there is an overshoot. Oxy-ped is limited to measurement and display and does not ensure the transmission of data to the doctor. It is also an essential service for home use. Indeed, in some cases, immediate feedback from the doctor is very necessary for the patient.

As long as these different products have had some success when they are put on the market, they still have a certain limitation in the services they offer, including, for example, the concept of mobility and real-time alert. In the next part, we will detail the design of our proposed intelligent medical bracelet (IMB) solution.

\section{GENERAL CONTEXTS OF THE IMB DESIGN}

In order to enrich the field of bio-telemetry, to ensure the spirit of remote diagnosis, to reduce patient discomfort and to improve their quality of life, we set ourselves the following objective in this study: Design a medical device (IMB) to monitor the vital signs (body temperature, heart rate, respiratory rate, blood pressure) of a sick baby, an infected child or an elderly person in order to be able to react in case clinical worsening.

As a starting point, we chose to study two vital signs: temperature and heart rate. In fact, temperature and heart rate are two very important signals and their exceeding of thresholds can cause harmful effects on the state of health of the person concerned:

- Fever can cause dehydration, a heart attack and even acidosis if it is too high $(+38 \mathrm{C})$. Acidosis can cause dizziness, loss of concentration, coma, and even death.

- Cardiac arrest

- Pericarditis: infection of the pericardium which can be caused by rheumatic fever.

\subsection{Target Population}

The target population concerned by the use of IMB is:

- Children: To ensure the child's good health easily and safely.

- The elderly and the disabled: Thanks to this device, the elderly and the disabled can surpass the continuous support by remaining "free". In case of emergency, the doctor and parents will be alerted in a timely manner.

- Pediatric services: Sometimes monitoring certain vital signs, such as temperature, is more important than exact measurement in separate intervals in children in pediatric services. Indeed, the device proposed in this work presents an effective solution to help the nursing staff to control, continuously and remotely, the vital signs of the patients.

\subsection{IMB functionalities}

Several functionalities are offered by the smart medical bracelet which we cite:

- Measurement of body temperature at all times.

- Measure heartbeats at all times.

- Display of the temperature value measured at all times.

- Display of number of beats measured at each instant.

- Triggering of three types of alerts: sound, light and an SMS to the controllers (the doctor, the parents in the case of babies and children...) In the event of the thresholds being exceeded by one of the vital signs.

The following figure 1 shows the operating process of the application in the event of exceeding the predefined limits 
before.

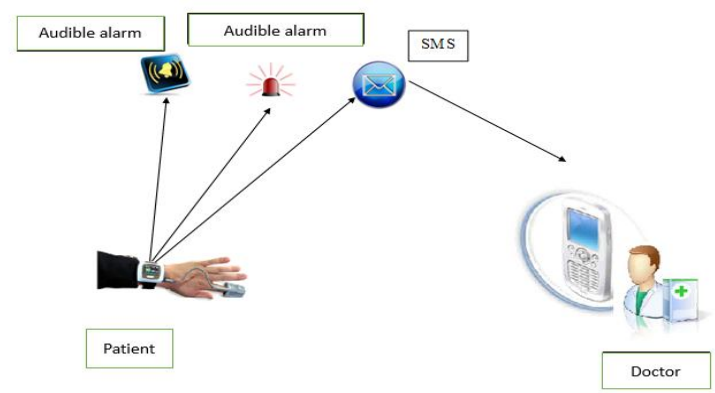

Figure 1: Application operating process if the predefined limits are exceeded before

\section{CONSTRAINTS FOR SETTING UP THE IMB}

When designing the IMB, several constraints were taken into account in order to satisfy a certain number of essential needs which meet the desired properties:

\subsection{Manufacturability}

All aspects related to the manufacturing of the final designed system should be considered. All the components necessary for the hardware implementation of the system should be available on the market and the final product should be compact in size.

\subsection{Cost}

The cost of developing the design should be considered and minimized with the best performance. In addition, the cost of the final product must be estimated. By choosing the components of this system, it was very difficult to find the most suitable transmission module that supports all the necessary functions at a suitable price.

\subsection{Energy}

Energy is a measure of the relationship between power consumed by the devices, and the rate at which useful work is done. The whole system must take into account the energy consumption which should be minimized as much as possible to maintain the long life of the intelligent medical bracelet (IMB) solution

\subsection{Health and safety}

Since this designed system is a medical device, the health and safety of the patient must be considered to avoid any dangers that may affect him by using the device. This is accomplished by ensuring that the patient is not exposed to unhealthy levels of electromagnetic radiation during wireless transmission. Also, the backup operation meets the conditions indicated in the ANSI Std standard. C95.1. The system supply does not exceed $5 \mathrm{~V}$, which is completely safe for the human body in dry conditions. An important point is that the final product must be subjected to rigorous laboratory tests to ensure that it is safe for use.
The designed system protects the patient's file by showing it only to authorized persons. Different encryption techniques will be applied to address the issue of security and to provide the highest level of confidentiality for patient information in all stages from signal extraction to final data storage.

\section{WASPMOTE DESCRIPTION}

In order to meet the above requirements, Libelium team decided to create a new device specially designed to work with low consumption modes and with a completely modular philosophy and that is how Waspmote was born. This latter is an open source wireless sensor platform specially focused on the implementation of low consumption modes to allow the sensor nodes ("motes") to be completely autonomous and battery powered, offering a variable lifetime between 1 and 5 years depending on the duty cycle and the radio used.

Waspmote Technical Guide is presented as follow:

- Microcontroller: ATmega1281

- Frequency: $14 \mathrm{MHz}$

- SRAM: 8KB

- EEPROM: 4KB (1KB reserved)

- FLASH: $128 \mathrm{~KB}$

- SD Card: 2GB

- Weight: 20gr

- Dimensions: 73.5 x 51 x $13 \mathrm{~mm}$

- Temperature Range: $\left[-10^{\circ} \mathrm{C},+65^{\circ} \mathrm{C}\right]$

The following figure 2 present the design of Waspmote.

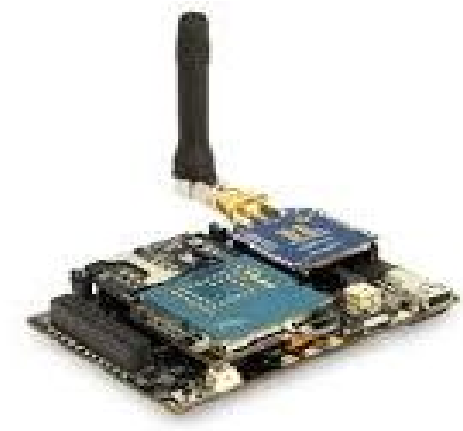

Figure 2: Waspmote 802.15.4 PRO SMA 2 DBI

To send information to the computer, a Waspmote Gateway are used. This device allows to collect data which flows through the sensor network into a PC or device with a standard USB port. Waspmote Gateway (figure 3 ) will act as a "data bridge or access point" between the sensor network and the receiving equipment. This receiving equipment will be responsible for storing and using the data received depending on the specific needs of the application. 


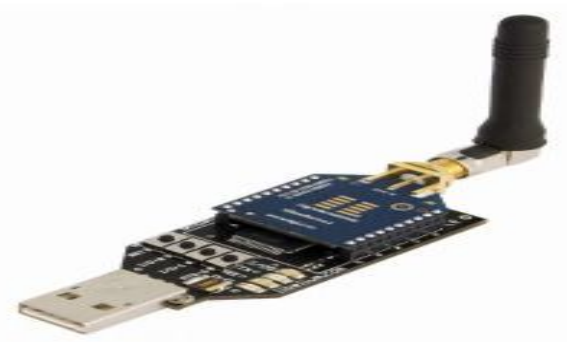

Figure 3: Waspmote Gateway 802.15.4 PRO SMA 2 DBI 6. DESIGN OF AN IMB PROTOTYPE

\subsection{Presentation of the platform used and the various equipment}

The prototype of the intelligent medical bracelet (IMB) is composed of the following list of component and shown by the figures:

- Waspmote 802.15.4 PRO SMA 2 DBI (Figure 2)

- Waspmote Gateway 802.15.4 PRO SMA 2 DBI(Figure 3)

- GPS module

- GSM / GPRS module

-Temperature sensor

- $\mathrm{SpO} 2$ sensor: for the measurement of heartbeats

- Basic LCD

- Green and red LED

The choice of this material was made following a comparative study of the various wireless sensor node platforms on the market. Waspmote has the advantage of being able to integrate different types of sensors and data communication modules with different protocols.

\subsection{Software implementation}

- Communication: in this phase, communication (figure 4) takes place between the Waspmote sensor and the Gateway. The sensor sends the packets containing the data such as temperature, heartbeat, etc. to the Gateway linked to the server which allows to receive this data via the XBee module by the 802.15.4 communication protocol.

- Database Server: named WaspmoteDB developed with Mysql-DataBase - java-5.1.15 for the management of the database used to save the data retrieved from the sensor and allowing access to this data remotely.

- Interface: presents the interface of the developed application.

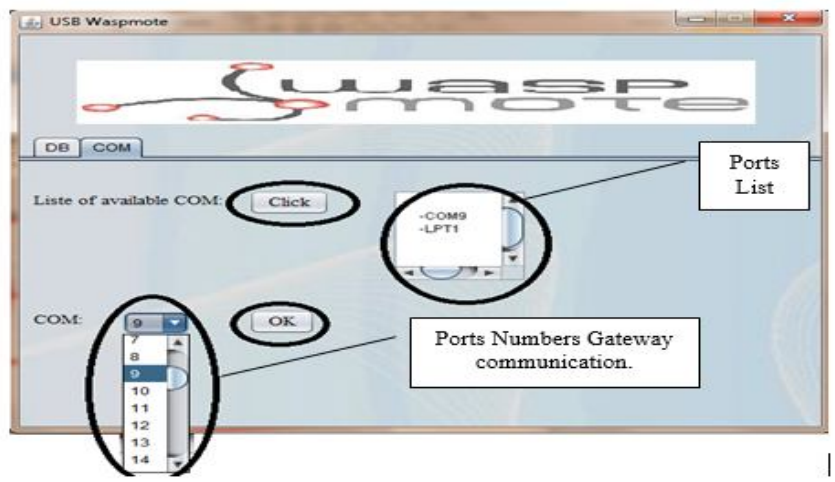

Figure 4: Communication interface between Sensor and Gateway
By running the application, we receive the data transmitted from the sensor to the Gateway in real time. As a result, we get the display of the packets sent by Waspmote to the Gateway. These data will then be saved in the server database as shown in Figure 5 below:

\begin{tabular}{|c|}
\hline USB Waspmote \\
\hline 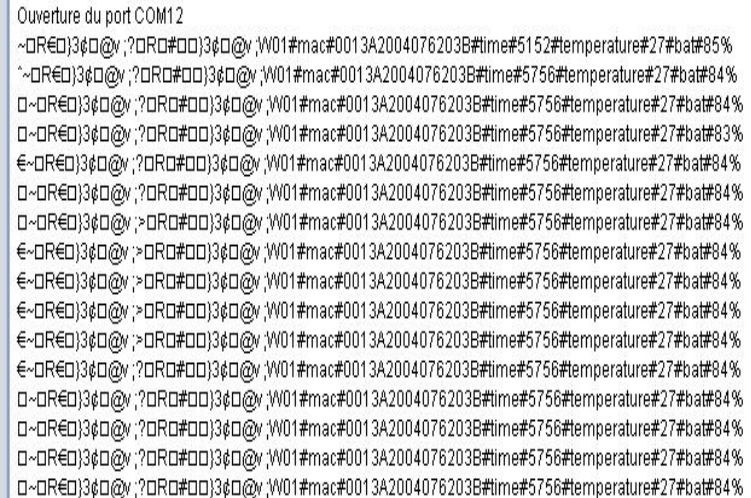 \\
\hline
\end{tabular}

Figure 5: Real-time display of packets received and saved in the database

\section{CONCLUSION}

In this paper, we presented the design of a prototype of a new generic RCSF platform for health called the Intelligent Medical Bracelet. We have detailed the various hardware components of this Bracelet as well as the software implementation of the communication protocol, of a database server as well as the interface of the developed application. This bracelet will allow the monitoring of patients' vital signs and the sending of alerts if they are exceeded. Work in progress on the Intelligent Medical Bracelet project will integrate other application aspects in order to enrich the number of services offered by the final product. Technology transfer to on-board systems is also being explored in order to be able to manufacture a Medical Bracelet that can meet the needs of the medical market.

\section{REFERENCES}

1. A Steib, JG Hentz, M Chauvin, JP Dupeyron, 2012. En ligne : http://www.sfar.org

2. Alzaheimer-medical-intelligence, 2007. COLUMBA. En ligne : http://www.actualites-news-environnement.com. I.F. AKYILDIZ, Weilian SU, Yogesh Sankarasubramaniam, and Erdal CAYIRCI, A servey on sensor networks, IEEE Communications Magazine, pp.102-114, August 2002. https://doi.org/10.1109/MCOM.2002.1024422

3. J. PIGNIEZ. 2008. COLUMBA, le bracelet anti-fugue de MEDICAL INTELLIGENCE. En ligne : http://www.gerontechnologie.net

4. Oxymètre de pouls OXY 6 pédiatrique, 2012. URL : http://www.doctorshop.fr. 
5. P. Huneault 2008. COLUMBA. En ligne: http://www.mdeie.gouv.qc.ca

6. R. Hatler, D. Gurganious, C. Chi. In Proceedsings of 2010 3rd International Symposium on Applied Sciences in Biomedical and Communication Technologies, Rome, Italy, November 2010.

7. S. Cypher, et al., "Prevailing over Wires in Healthcare Environments: Benefits and Challenges" IEEE Communications Magazine, pp. 56-63, April 2006. https://doi.org/10.1109/MCOM.2006.1632650

8. S. Pacelli, et al., "Sensing Fabrics for Monitoring Physiological and Biomechanical Variables: E-textile solutions" 3rd IEEE-EMBS International Summer School and Symposium on Medical Devices and Biosensors, Boston, September, 2006. https://doi.org/10.1109/ISSMDBS.2006.360082

9. T. Jovanov, et al., "A wireless body area network of intelligent motion sensors for computer assisted physical rehabilitation" Journal of NeuroEngineering and Rehabilitation, March 2005.

10. V. Shnayder, et al., "Sensor Networks for Medical Care" Technical Report TR-08-05, Division of Engineering and Applied Sciences, Harvard University, 2005.

11. V, Megalooikonomou, et al., "Medical Data Fusion for Telemedicine" IEEE Engineering in Medicine and Biology Magazine, pp. 36-42, September/October 2007. https://doi.org/10.1109/EMB.2007.901790

12. Tauhidur Rahman, Alexander Travis Adams, Mi Zhang, Erin Cherry, Bobby Zhou, Huaishu Peng, and Tanzeem Choudhury. Bodybeat: a mobile system for sensing non-speech body sounds. In MobiSys, volume 14, pages 2-13, 2014.

13. Xiao Sun, Zongqing Lu, Wenjie Hu, and Guohong Cao. Symdetector: detecting soundrelated respiratory symptoms using smartphones. In Proceedings of the 2015 ACM International Joint Conference on Pervasive and Ubiquitous Computing, pages 97\{108. ACM, 2015.

14. Worldometers.info, "Coronavirus Update: March 30, 2020." Retrived from

https://www.worldometers.info/coronavirus/ on March 30, 2020, pp. 1-2, 2020.

15. A. I. Howlader and M. A. Islam, "Information-seeking behaviour of undergraduate students: A developing country perspective," IFLA J., vol. 45, no. 2, pp. 140-156, Jun. 2019. https://doi.org/10.1177/0340035219842312

16. A. Bansal et al., "Information Seeking and Satisfaction with Information Sources Among Spouses of Men with Newly Diagnosed Local-Stage Prostate Cancer," J. Cancer Educ., vol. 33, no. 2, pp. 325-331, 2018.

17. Y. Qi, C. D. Du, T. Liu, X. Zhao, and C. Dong, "Experts' conservative judgment and containment of COVID-19 in early outbreak," J. Chinese Gov., vol. 2346, pp. 1-20, Mar. 2020.

18. K. Sun, J. Chen, and C. Viboud, "Early epidemiological analysis of the coronavirus disease 2019 outbreak based on crowdsourced data: a population-level observational study," Lancet Digit. Heal., vol. 2, no. 4, pp. e201-e208, Apr. 2020.

https://doi.org/10.1016/S2589-7500(20)30026-1

19. A. J. Chetwynd, K. E. Wheeler, and I. Lynch, "Best practice in reporting corona studies: Minimum information about Nanomaterial Biocorona Experiments (MINBE)," Nano Today, vol. 28, p. 100758, Oct. 2019.

20. T. Ahmad and J. Hui, "One Health approach and Coronavirus Disease 2019," Hum. Vaccin. Immunother., vol. 00, no. 00, pp. 1-2, Mar. 2020.

21. E. Gu and L. Li, "Crippled community governance and suppressed scientific/professional communities: a critical assessment of failed early warning for the COVID-19 outbreak in China," J. Chinese Gov., vol. 0, no. 0, pp. 1-18, Mar. 2020.

22. G. L. Alexander et al., "Advancing health information technology roadmaps in long term care," Int. J. Med. Inform., vol. 136, no. January, p. 104088, Apr. 2020. https://doi.org/10.1016/j.ijmedinf.2020.104088

23. A. J. Linn et al., "Patients' Online Information-Seeking Behavior Throughout Treatment: The Impact on Medication Beliefs and Medication Adherence," Health Commun., vol. 34, no. 12, pp. 1461-1468, Oct. 2019.

24. L. D. Sherman, M. S. Patterson, A. Tomar, and L. T. Wigfall, "Use of Digital Health Information for Health Information Seeking Among Men Living With Chronic Disease: Data From the Health Information National Trends Survey,” Am. J. Mens. Health, vol. 14, no. 1, p. 155798832090137 , Jan. 2020.

25. I. Khaleel et al., "Health information overload among health consumers: A scoping review," Patient Educ. Couns., vol. 103, no. 1, pp. 15-32, Jan. 2020. https://doi.org/10.1016/j.pec.2019.08.008

26. N. Diviani, C. Zanini, R. Jaks, M. Brach, A. Gemperli, and S. Rubinelli, "Information seeking behavior and perceived health literacy of family caregivers of persons living with a chronic condition. The case of spinal cord injury in Switzerland," Patient Educ. Couns., no. 2019, 2020.

27. M. Shim and H. S. Jo, "What quality factors matter in enhancing the perceived benefits of online health information sites? Application of the updated DeLone and McLean Information Systems Success Model," Int. J. Med. Inform., vol. 137, no. January, p. 104093, May 2020.

28. P. Wenjing, S. Shijie, and Zhang Yan, "Consumer health information needs: A systematic review of measures," Inf. Process. Manag., vol. 57, no. 2, p. 102077, Mar. 2020.

https://doi.org/10.1016/j.ipm.2019.102077 